\title{
Signs of Preclinical Wernicke's Encephalopathy and Thiamine Levels as Predictors of Neuropsychological Deficits in Alcoholism without Korsakoff's Syndrome
}

\author{
Anne-Lise Pitel', Natalie M Zahr',2, Karen Jackson², Stephanie A Sassoon ${ }^{2}$, Margaret J Rosenbloom ${ }^{1,2}$, \\ Adolf Pfefferbaum ${ }^{1,2}$ and Edith V Sullivan*, I \\ 'Department of Psychiatry and Behavioral Sciences, Stanford University School of Medicine, Stanford, CA, USA; ${ }^{2}$ Neuroscience Program, \\ SRI International, Menlo Park, CA, USA
}

\begin{abstract}
The purpose of this study was to determine whether meeting historical criteria for unsuspected Wernicke's encephalopathy (WE), largely under-diagnosed in vivo, explains why some alcoholics have severe neuropsychological deficits, whereas others, with a similar drinking history, exhibit preserved performance. Demographic, clinical, alcohol related, and neuropsychological measures were collected in 56 abstinent alcoholics and 38 non-alcohol-dependent volunteers. Alcoholics were classified using the clinical criteria established by Caine et al (1997) and validated in their neuropathological study of alcoholic cases. Our alcoholics who met a single criterion were considered 'at risk for WE' and those with two or more criteria with 'signs of WE'. Whole blood thiamine was also measured in 22 of the comparison group and 28 alcoholics. Of the alcoholics examined, $27 \%$ met no criteria, 57\% were at risk for WE, and $16 \%$ had signs of WE. Neuropsychological performance of the alcoholic subgroups was graded, with those meeting zero criteria not differing from controls, those meeting one criterion presenting mild-to-moderate deficits on some of the functional domains, and those meeting two or more criteria having the most severe deficits on each of the domains examined. Thiamine levels were selectively related to memory performance in the alcoholics. Preclinical signs of WE can be diagnosed in vivo, enabling the identification of ostensibly 'uncomplicated' alcoholics who are at risk for neuropsychological complications. The graded effects in neuropsychological performance suggest that the presence of signs of WE explains, at least partially, the heterogeneity of alcoholism-related cognitive and motor deficits. Neuropsychopharmacology (20II) 36, 580-588; doi:I0.1038/npp.2010.189; published online 20 October 2010
\end{abstract}

Keywords: alcoholism; Wernicke's encephalopathy; neuropsychology; thiamine; Korsakoff's syndrome; memory

\section{INTRODUCTION}

In 2000, alcohol consumption was responsible for 85000 deaths in the United States (Cargiulo, 2007), with the insidious effects of alcohol abuse and dependence being responsible for $46 \%$ of them (http://www.cdc.gov/mmwr/ preview/mmwrhtml/mm5337a2.htm). Indeed, chronic heavy drinking is associated with a high risk of developing acute and chronic physical and mental health problems, including alcohol-related neuropsychiatric disorders and neuropsychological dysfunction that we speculate are related to neuropathology of preclinical

*Correspondence: Dr EV Sullivan, Department of Psychiatry and Behavioral Sciences, Stanford University School of Medicine (MC5723), 401 Quarry Road, Stanford, CA 94305-5723, USA, Tel: + I 650498 7328, Fax: + I 650859 2743, E-mail: edie@stanford.edu Received 27 July 2010; revised 20 August 2010; accepted 13 September 2010
Wernicke's encephalopathy (WE; Harper, 2009; Sullivan and Pfefferbaum, 2009).

WE is an acute reaction to thiamine (vitamin B1) deficiency characterized by the classical clinical triad of confusion, ataxia, and oculomotor abnormalities, including nystagmus and ophthalmoplegia (Wernicke, 1881). Severe or insufficiently treated cases of thiamine deficiency can result in enduring amnesia and ataxia defining Korsakoffs Syndrome (KS; Kopelman et al, 2009). By far the most common cause of thiamine deficiency throughout the world is alcoholism (Thomson, 2000). Low circulating levels of thiamine have been reported in $30-80 \%$ of alcoholic patients (Thomson et al, 1987), with considerable variability in individual vulnerability for developing thiamine deficiency (Thomson and Marshall, 2006). Alcoholics are at special risk for thiamine deficiency because of poor diet (associated with malnutrition), compromised thiamine absorption from the gastrointestinal tract, impaired thiamine storage, and reduced thiamine phosphorylation (Thomson, 2000). Circulating levels of thiamine per se, 
however, may not be an accurate reflection of thiamine concentrations. In fact, $80 \%$ of thiamine is stored in the form of thiamine diphosphate (TDP), which is the active molecule used in the metabolic pathways of glucose metabolism (Guerrini et al, 2009).

Mild-to-moderate cognitive and motor deficits have been reported in alcoholics without apparent neuropsychiatric complications (ie uncomplicated alcoholics). These alcoholrelated neuropsychological deficits comprise episodic memory (Pitel et al, 2007a), working memory and executive functions (Noel et al, 2001), visuoconstruction abilities (Dawson and Grant, 2000), and upper and lower limb motor skills (Sullivan et al, 2000b). A continuum of cognitive impairments has been proposed (Parsons, 1998; Ryback, 1971), with KS performing worse than uncomplicated alcoholics. Nonetheless, these groups can overlap, with the most poorly performing alcoholics without KS scoring comparably to the best performing alcoholics with KS (Pitel et al, 2008). According to the original continuity hypothesis (Ryback, 1971), the main contributing factor of neuropsychological performance in alcoholics is the amount of alcohol consumed over a lifetime. To date, however, correlations between cognitive and motor results and drinking history are typically inconsistent (Noel et al, 2001). It remains unknown why some alcoholics exhibit severe neuropsychological deficits as well as compromised health status, whereas others with ostensibly similar overall alcohol consumption levels appear to drink with some impunity, do not seek treatment, and are seldom the focus of scientific investigations (Fein and Landman, 2005).

Postmortem studies of large samples of alcoholic cases have concluded that WE is under-diagnosed in vivo (Harper, 2006; Torvik et al, 1982), resulting in heightened risks for development of KS (85\% of WE survivors; Day et $a l, 2008)$ or premature death $(20 \%$ in postmortem identification of WE; Harper et al, 1986). Under-diagnosis of WE could be related to the fact that current criteria require the full clinical triad of symptoms. Yet, all three clinical symptoms were identified from chart review in only $16 \%$ of patients identified with postmortem neuropathological WE signs of mammillary body and thalamic atrophy (Harper et al, 1986; Naidoo et al, 1991). To assist in retrospective diagnosis of WE and KS, Caine et al (1997) proposed 'operational criteria' for identifying WE events postmortem from chart review of clinical histories of alcoholic cases. Definitive diagnosis of WE was determined neuropathologically and was associated in vivo with the presence of two of the following four criteria: (1) history of dietary deficiencies, (2) oculomotor abnormalities, (3) cerebellar dysfunction, and (4) either an altered mental state or mild-memory impairment. The use of these operational criteria suggested that the antemortem identification of WE could be achieved with a high degree of specificity. Despite the utility of these retrospectively applied criteria (Baker et al, 1999), diagnosis of WE could be further enhanced by applying these criteria during life and supplementing them with targeted and quantitative neurological and neuropsychological examinations (Sechi and Serra, 2007).

The goals of this study were (1) to examine whether the application of the Caine et al chart-review criteria during life enables identification of alcoholic subgroups at risk for or with signs of WE, and (2) to determine whether the presence of risk for or signs of WE contributes to the heterogeneity in the profile of alcohol-related cognitive and motor impairments measured quantitatively.

\section{MATERIALS AND METHODS}

\section{Participants}

The participants were 56 alcoholics and 38 healthy non-alcoholic comparison subjects. All participants were volunteers and, after the procedures had been fully explained, gave written informed consent obtained according to institutional review board guidelines of SRI International and Stanford University School of Medicine to participate in this study. They were paid a modest stipend for participation.

All participants were administered the Structured Clinical Interview for DSM-IV-TR (SCID; First et al, 1995) by a clinical psychologist or a research nurse trained and calibrated with one another. Alcohol dependence was determined by DSM-IV criteria. Exclusionary criteria for all subjects were the following: presence of other DSM-IV Axis I diagnoses including schizophrenia, bipolar disorder, attention deficit hyperactivity disorder, and post-traumatic stress disorder (mood disorders other than bipolar were not exclusionary for the alcoholic group); history of central nervous system trauma, such as loss of consciousness for $>30 \mathrm{~min}$, seizures not related to alcohol withdrawal, or degenerative disease; or serious medical conditions, such as severe endocrine, hepatic, or renal disease. None of the participants had KS as defined by the DSM-IV criteria for Alcohol-Induced Persisting Amnestic Disorder. The alcoholic group comprised 41 men and 15 women recruited from local rehabilitation centers. The healthy control group comprised 20 men and 18 women recruited by Internet and newspaper advertisements, community flyers, and word of mouth.

Table 1 provides demographic data for each group of subjects. Two sample $t$-tests indicated that the groups did not differ significantly with respect to age $(t(92)=-0.19$, $p=0.85)$ or body mass index (BMI) $(t(92)=-0.13$, $p=0.90)$, but the alcoholic group had fewer years of formal education $(t(92)=-3.84, p=0.001)$ than controls. Furthermore, the alcoholic group had more men than women relative to the sex ratio in the control group $\left(\chi^{2}=3.8\right.$, $p=0.05)$.

\section{Sorting of Alcoholics Using 'Caine Criteria'}

According to Caine et al (1997), alcoholics with at least two of the four signs described below were considered to have 'signs of WE'. In this study, these criteria were extended to classify individuals with only one criterion as 'at risk for WE'.

Dietary deficiency. This deficiency was quantified according to the information collected along with a detailed lifetime alcohol history obtained by the SCID interviewer. Subjects were asked whether meals were missed because of drinking, and if so, how many days of missed meals per drinking episode and how frequently this occurred. 
Table I Demographic, Clinical, and Alcohol-Related Variables in the Control Group, the Total Alcoholic Group and the three Subgroups of Alcoholics (Mean $\pm \mathrm{SD}$ )

\begin{tabular}{|c|c|c|c|c|c|c|c|c|c|c|c|c|c|}
\hline \multirow[t]{3}{*}{ Variables } & & \multicolumn{4}{|c|}{ Control subjects } & \multicolumn{6}{|c|}{ Alcoholic subjects } & \multirow[t]{3}{*}{ F-value (dF) } & \multirow{3}{*}{$\begin{array}{l}\text { Between-groups } \\
\text { comparisons }\end{array}$} \\
\hline & & \multicolumn{2}{|c|}{$\begin{array}{l}\text { Total group } \\
\quad(N=38)\end{array}$} & \multicolumn{2}{|c|}{$\begin{array}{l}\text { Total group } \\
(N=56)\end{array}$} & \multicolumn{2}{|c|}{$\begin{array}{l}0 \text { criteria } \\
(N=15)\end{array}$} & \multicolumn{2}{|c|}{$\begin{array}{l}\text { I criterion } \\
(N=32)\end{array}$} & \multicolumn{2}{|c|}{$\begin{aligned} \geqslant & 2 \text { criteria } \\
& (N=9)\end{aligned}$} & & \\
\hline & & Mean & SD & Mean & SD & Mean & SD & Mean & SD & Mean & SD & & \\
\hline Age & & 46.83 & 15.12 & 46.29 & 11.45 & 47.72 & 12.98 & 44.50 & 11.3 & 50.29 & 8.79 & $F(3,94)=0.55$ & Ctrl $=0$ crit $=1$ crit $=2$ crit \\
\hline Years of education & & 15.26 & 2.6 & 13.38 & 2.15 & 14.53 & 2.17 & 13.10 & 2.16 & 12.88 & 1.69 & $F(3,94)=5.53^{*}$ & $(C t r \mid=0$ crit $)>(\mid$ crit $=2$ crit $)$ \\
\hline BMI & & 26.94 & 5.61 & 26.81 & 4.24 & 25.06 & 4.65 & 27.23 & 4.27 & 27.33 & 4.27 & $F(3,94)=0.42$ & Ctrl $=0$ crit $=1$ crit $=2$ crit \\
\hline AUDIT & & 1.58 & 2.11 & 20.04 & 14.94 & 16.27 & 14.22 & 23.28 & 15.07 & 14.89 & 14.04 & $F(3,77)=20.19 *$ & Ctrl $\mid<(0$ crit $=\mid$ crit $=2$ crit $)$ \\
\hline SADDQ & & 1.05 & 2.06 & 17.39 & 12.4 & 17.53 & $12.9 \mid$ & 18.87 & 12.46 & 11.88 & 10.99 & $F(3,79)=29.32^{*}$ & Ctrl $<($ Ccrit $=\mid$ crit $=2$ crit $)$ \\
\hline Lifetime alcohol intake (kg) & & 36.7 & 79.16 & | 149.00 & 956.63 & 1036.43 & 1121.82 & 1113.53 & 896.62 & | 462.74 & $9|3.5|$ & $F(3,94)=17.67 *$ & Ctr $\mid<(0$ crit $=\mid$ crit $=2$ crit $)$ \\
\hline Sobriety (days) & & NA & NA & 315.90 & 592.08 & 353.27 & 590.95 & 333.78 & 665.41 & 190.00 & 248.08 & $F(2,56)=0.24$ & 0 crit $=1$ crit $=2$ crit \\
\hline \multirow{2}{*}{$\begin{array}{l}\text { Time since last met criteria for } \\
\text { alcohol dependency (weeks) }\end{array}$} & & NA & NA & 66.60 & 109.77 & 104.20 & 149.96 & 60.41 & 98.68 & 25.97 & 33.01 & $F(2,56)=1.58$ & 0 crit $=1$ crit $=2$ crit \\
\hline & & $N$ & $\%$ & $\mathbf{N}$ & $\%$ & $N$ & $\%$ & $N$ & $\%$ & $N$ & $\%$ & $\chi^{2}$ value $(\mathrm{dF})$ & $\begin{array}{l}\text { Between-groups } \\
\text { comparisons }\end{array}$ \\
\hline \multirow{2}{*}{$\begin{array}{l}\text { Physiological symptoms } \\
\text { of dependence }\end{array}$} & Tolerance & NA & NA & 43 & $77 \%$ & 8 & $53 \%$ & 28 & $88 \%$ & 7 & $78 \%$ & $\chi^{2}(2)=8.90^{*}$ & Ocrit < (I crit = 2crit $)$ \\
\hline & Withdrawal & NA & NA & 40 & $71 \%$ & 7 & $47 \%$ & 26 & $81 \%$ & 7 & $78 \%$ & $\chi^{2}(2)=10.32 *$ & Ocrit $<($ I crit $=2$ crit $)$ \\
\hline
\end{tabular}

Abbreviations: BMI, body mass index; AUDIT, alcohol use disorders identification test; SADDQ, short alcohol dependence data questionnaire.

Ocrit: alcoholics meeting none of the criteria.

I crit: alcoholics meeting one of the criterion, at risk for WE.

2 crit: alcoholics meeting at least two of the criteria, with signs of WE.

Ctrl: control subjects.

*Significant effect of group $(p<0.05)$.

An individual reporting at least 30 missed-meal days over their lifetime was considered to have potentially experienced dietary deficiency, albeit not necessarily thiamine deficiency or depletion.

Oculomotor abnormalities. These abnormalities were determined during a neurological examination. Saccades and smooth pursuit were observed during frontal eye gaze and fixed head position while subjects tracked the examiner's index finger as it moved to the extreme right, extreme left, upward, and downward. An individual with nystagmus was considered to have an oculomotor abnormality.

Cerebellar dysfunction. This dysfunction was defined by instability of gait and static posture, which were assessed using the eyes open condition of the Fregly-Graybiel walka-line test (Fregly et al, 1973). Performance on this ataxia test was normalized to age and sex derived from a separate age regression analysis for 108 healthy men and 87 healthy women (data taken from Sullivan et al, 2010). Cutoff scores were defined as the lower limits of the $90 \%$ confidence interval. An individual with performance on two or more ataxia test parts with scores below sex- and age-corrected scores was considered to have ataxia, a possible reflection of cerebellar dysfunction (cf., Sullivan et al, 2000a).

Altered mental state or mild-cognitive impairment. Altered mental state or mild general cognitive impairment was assessed using the Dementia Rating Scale (DRS; Mattis,
1988). An individual with a total score equal to or below the published cutoff score of 123 out of 144 was considered to have altered mental state or mild-cognitive impairment.

\section{Interview and Questionnaires}

Measures of the severity of alcohol dependence were collected with two questionnaires (the Alcohol Use Disorders Identification Test, AUDIT; Saunders et al, 1993; and the Short Alcohol Dependence Data Questionnaire, SADDQ; Raistrick et al, 1983) and questions accompanying the SCID regarding time since last met criteria for alcohol dependency (in weeks), length of sobriety (in days), and presence of physiological symptoms of dependency (tolerance and/or withdrawal). Lifetime alcohol consumption (in $\mathrm{kg}$ ) was quantified using a modification of a semi-structured timeline interview (Skinner, 1982).

\section{Neuropsychological Tests}

Two tests quantified IQ: the National Adult Reading Test (NART; Nelson, 1982) provided an estimate of premorbid IQ, and the Matrix Reasoning Subtest of the WAIS III (Wechsler, 2001) estimated current performance IQ. Subjects completed the Wechsler Memory Scale-Revised (WMS-R; Wechsler, 1987), which yielded five indices related to memory. We used the Trail Making Test (Lezak, 1995) and two tests of word production (semantic fluency and letter fluency) to evaluate executive functions. Visuoconstruction abilities were assessed using the Rey-Osterrieth 
Complex Figure Test (Osterrieth and Rey, 1944; Rey, 1942). Standard administration of the digit symbol subtest of the WAIS-R (Wechsler, 1981) evaluated psychomotor speed, and upper motor limb skills were examined using the fine finger movement test (Corkin et al, 1986) and the grooved pegboard test (Matthews and Kleve, 1964).

\section{Assessment of Thiamine in Whole Blood}

Whole blood was collected from 28 alcohol-dependent and 22 control participants. Frozen specimens were transported to ARUP laboratories (Salt Lake City, Utah, http://www. aruplab.com) for assessment of TDP in whole blood. Approximately $80 \%$ of the total thiamine content of whole blood is present as TDP, the biologically active form of thiamine; thus, whole blood TDP measures provide a good indication of the adequacy of thiamine body stores (Mancinelli et al, 2003).

\section{Statistical Analysis}

Alcoholic and control subjects were compared on the clinical and alcohol-related variables using $t$-tests and on six neuropsychological domains using repeated measures ANOVA. As the two groups differed in education, we also conducted an ANCOVA using years of formal education as a covariate. Between-groups comparisons were then conducted using follow-up $t$-tests. Once classified according to Caine et al criteria, the subgroups of alcoholics were compared with controls and each other on the clinical and alcohol-related variables using one-way ANOVAs and on the six neuropsychological domains using repeated measures ANOVA and then ANCOVA using the demographic, clinical, and alcohol-related variables that differed among the alcoholic subgroups as covariates. Between-groups comparisons were conducted using follow-up $t$-tests. Owing to the relatively small sample size of one of the subgroups of alcoholics (those with signs of $\mathrm{WE}$ ), non-parametric tests (Mann-Whitney $U$-tests) were used to verify the parametric results. Furthermore, because of the potentially confounding effects of education, we examined in more detail premorbid and current IQ by comparing controls and the three subgroups of alcoholics using a repeated-measured ANOVA and follow-up $t$-tests. Then, we compared TDP levels in alcoholics classified as having had dietary deficiencies with those without this history, and examined in alcoholics the relationships between TDP levels, drinking history, and age using Pearson's correlations. Finally, we examined the relationships between neuropsychological performance and TDP levels in the whole group of alcoholics using a multiple regression.

As no sex differences were identified in neuropsychological performance in the control and alcoholic groups, data for men and women were pooled for constructing standardized scores. The first step in constructing composite scores by functional domain involved transforming each neuropsychological test score for each subject into a standardized $\mathrm{z}$-score based on the mean performance of the control group. The sign of all variables for which high scores were in the impaired direction (such as completion time or number of errors) was reversed, so that all the z-scores had the same direction: the higher the z-score, the better the performance. Thus, all cognitive and motor variables were on the same scale. The $\mathrm{z}$-scores were then aggregated to reflect six domains: (1) verbal (premorbid) and performance (current) IQ (mean of NART and Matrix Reasoning), (2) memory (mean of the five WMS-R indices), (3) executive functions (mean of the completion time on the Trail Making B Test and the two fluency scores), (4) visuoconstruction (Complex Figure copy score), (5) processing speed (digit symbol completion time), and (6) upper limb motor skills (mean of fine finger movement and grooved pegboard scores). Concerning the analysis of the IQ results, we used age- and education-corrected z-scores for the NART score and the WAIS III Matrix Reasoning subtest score.

\section{RESULTS}

\section{Comparisons between Controls and Alcoholics}

Clinical and alcohol-related variables. Means and SD of clinical and alcohol-related variables are provided in Table 1. Alcoholics had higher scores on the AUDIT $(t(92)=-7.54, p \leqslant 0.001)$ and the SADDQ $(t(92)=-8.01$, $p \leqslant 0.001)$ and consumed $\sim 30$ times more alcohol over their lifetime $(t(92)=7.14, p \leqslant 0.001)$ than controls.

Neuropsychological test composite scores. Repeated measures ANOVA for two groups and six neuropsychological domains revealed significant effects of group $(\mathrm{F}(1,77)=9.32$; $p=0.003)$, neuropsychological domains $(\mathrm{F}(5,385)=6.13$; $p<0.001)$, and their interaction $(\mathrm{F}(5,385)=2.84 ; p=0.016)$. With education as a covariate, ANCOVA showed a significant effect of education $(\mathrm{F}(1,76)=8.55 ; p=0.005)$ and persisting effects of group $(\mathrm{F}(1,76)=3.85 ; p=0.05)$ and neuropsychological domains $(\mathrm{F}(5,380)=5.15 ; p<0.001)$. The interaction was no longer significant $(\mathrm{F}(5,380)=1.22 ; p=0.30)$. Alcoholics achieved lower scores than controls on measures of IQ $(t(77)=3.39, p=0.001)$, memory $(t(78)=3.40, p=0.001)$, and processing speed $(t(91)=3.05, p=0.003)$, and showed a trend on executive functions $(t(90)=1.80, p=0.07)$ and visuoconstruction $(t(92)=1.76, p=0.08)$. The two groups did not differ, however, in performance on tests of upper limb motor skills $(t(91)=1.19, p=0.24)$ (Figure 1).

\section{Classification According to Caine et al Criteria}

Classification of the alcoholics by the Caine et al criteria revealed that $9(16 \%)$ had signs of WE (two or more criteria), 32 (57\%) were at risk of WE (one criterion), and $15(27 \%)$ met no criterion (Table 2). One alcoholic met three criteria, and none had all four. Dietary deficiency was noted in $52 \%$ of the alcoholics and ataxia in $36 \%$. Only two alcoholics had evidence of nystagmus, and none had mildcognitive deficits according to the criteria described herein (ie DRS score $\leqslant 123$ ). In the control group, one woman met the criteria for ataxia.

The subgroups were indistinguishable with respect to age, sex, and BMI (Table 1). However, alcoholics who did not meet any of WE criteria were more educated than those at risk $(t(45)=2.13, p=0.04)$ and tended to be more educated 


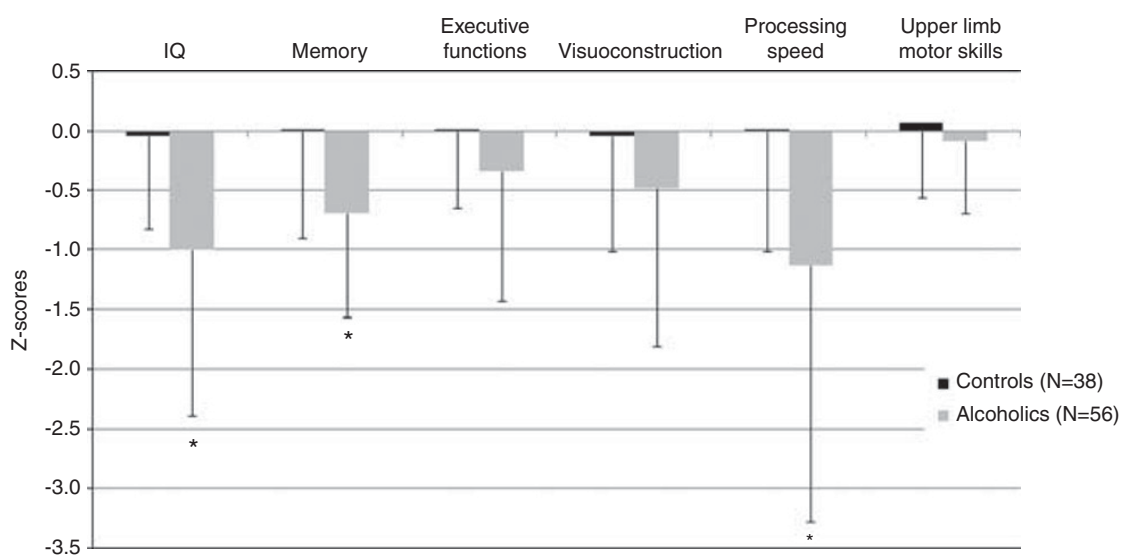

Figure I Neuropsychological performance in the control group, and the total alcoholic group (mean \pm SD). *Significant difference compared with controls $(p<0.05)$.

Table 2 Classification of Alcoholics into Subgroups According to the Caine et al Criteria

\begin{tabular}{|c|c|c|c|c|c|c|c|c|c|c|c|c|c|c|c|}
\hline \multirow[t]{3}{*}{ Groups } & \multirow{3}{*}{$\begin{array}{c}\text { Total } \\
N\end{array}$} & \multicolumn{8}{|c|}{ Criteria } & \multicolumn{6}{|c|}{ Subgroups } \\
\hline & & \multicolumn{2}{|c|}{$\begin{array}{l}\text { Dietary } \\
\text { deficiency }\end{array}$} & \multicolumn{2}{|c|}{ Nystagmus } & \multicolumn{2}{|c|}{ Ataxia } & \multicolumn{2}{|c|}{$\begin{array}{c}\text { Mild } \\
\text { cognitive deficit }\end{array}$} & \multicolumn{2}{|c|}{0 criteria } & \multicolumn{2}{|c|}{$\begin{array}{l}\text { I criterion: } \\
\text { at risk for WE }\end{array}$} & \multicolumn{2}{|c|}{$\begin{array}{l}\geqslant 2 \text { criteria: } \\
\text { signs of } W E\end{array}$} \\
\hline & & $\mathbf{N}$ & $\%$ & $N$ & $\%$ & $N$ & $\%$ & $\mathbf{N}$ & $\%$ & $N$ & $\%$ & $\mathbf{N}$ & $\%$ & $N$ & $\%$ \\
\hline Controls & 38 & 0 & 0.00 & 0 & 0.00 & 1 & 2.63 & 0 & 0.00 & 37 & 97.37 & 1 & 2.63 & 0 & 0.00 \\
\hline Control women & 18 & 0 & 0.00 & 0 & 0.00 & I & 5.56 & 0 & 0.00 & 17 & 94.44 & । & 5.56 & 0 & 0.00 \\
\hline Alcoholics & 56 & 29 & 51.78 & 2 & 3.57 & 20 & 35.71 & 0 & 0.00 & 15 & 26.79 & 32 & 57.14 & 9 & 16.07 \\
\hline Alcoholic men & 41 & 24 & 58.54 & 2 & 4.88 & 15 & 36.59 & 0 & 0.00 & 8 & 19.51 & 26 & 63.41 & 7 & 17.07 \\
\hline Alcoholic women & 15 & 5 & 33.33 & 0 & 0.00 & 5 & 33.33 & 0 & 0.00 & 7 & 46.67 & 6 & 40.00 & 2 & 13.33 \\
\hline
\end{tabular}

than those with signs of WE $(t(22)=2.63, p=0.07)(p \leqslant 0.07$ with non-parametric Mann-Whitney $U$-test). Alcoholics with history of dietary deficiency were younger than those without this history $(t(54)=-2.83, p=0.006)$ and dietary deficiency was observed more frequently in men than women $\left(\chi^{2}=11.17, p<0.001\right)$. Alcoholics with ataxia were older than those without ataxia $(t(54)=-3.59, p<0.001)$ and this criterion was more frequently noted in men than women $\left(\chi^{2}=4.05, p=0.04\right)$.

\section{Comparisons between Controls and Subgroups of Alcoholics}

Clinical and alcohol-related variables. One-way ANOVAs revealed a significant group effect (control group $v s$ alcoholic subgroups) on the AUDIT, SADDQ, and lifetime alcohol consumption. Among the alcoholic subgroups, however, there was no significant difference in length of sobriety or time since last meeting criteria for alcohol dependency (Table 1).

Follow-up $t$-tests showed that all three subgroups of alcoholics had higher scores than controls on the AUDIT and the SADDQ $(p<0.001$ in each case; Table 1$)$, but did not differ from each other $(p>0.10$ in each case). All three subgroups also had consumed more alcohol over their lifetime than controls $(p<0.001$ in each case). However, they did not differ from each other on lifetime alcohol consumption, length of sobriety, or time since last met criteria for alcoholism ( $p>0.10$ in each case). Compared with those who did not meet any criteria, alcoholics at risk for WE (one criterion) or with signs of WE (two or more criteria) were more likely to have developed tolerance (at risk: $\left(\chi^{2}=8.69, p=0.003\right)$; with signs: $\left(\chi^{2}=4.77\right.$, $p=0.03)$ ) and to have exhibited withdrawal symptoms (at risk: $\left(\chi^{2}=9.03, p=0.003\right)$; with signs: $\left.\left(\chi^{2}=7.69, p=0.005\right)\right)$.

Neuropsychological test composite scores. Repeated measures ANOVA for four groups (control group and three alcoholic subgroups) and six neuropsychological domains revealed significant effects of group $(\mathrm{F}(3,75)=12.41$; $p<0.001)$, neuropsychological domains $(\mathrm{F}(5,375)=11.61$; $p<0.001)$, and their interaction $(\mathrm{F}(15,385)=3.69 ; p<0.001)$ (Figure 2). After controlling for education, a four group ANCOVA showed a significant effect of education $(\mathrm{F}(1,74)=4.43 ; p=0.04)$ and persisting effects of group $(\mathrm{F}(3,74)=8.29 ; \quad p=0.001)$, neuropsychological domains $(\mathrm{F}(5,370)=4.21 ; p=0.001)$, and interaction $(\mathrm{F}(15,370)=$ 2.77; $p<0.001)$. 


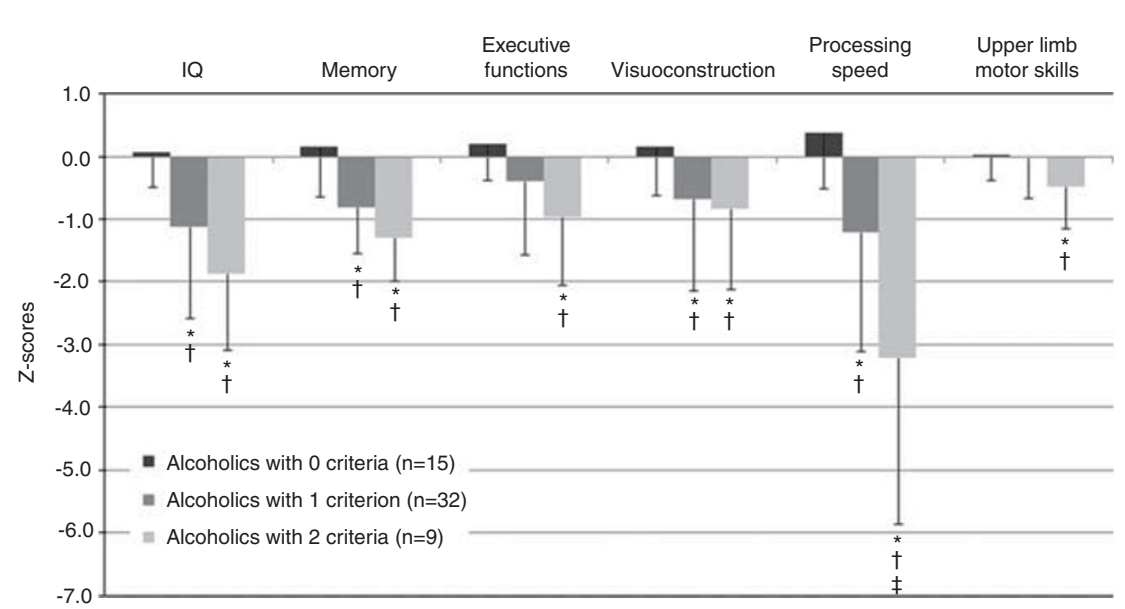

Figure 2 Neuropsychological performance in the three subgroups of alcoholics (mean $\pm S D$ ). *Significant difference compared with controls ( $p<0.05$ ). †Significant difference compared with alcoholics who did not meet any criteria $(p<0.05)$. ${ }^{\ddagger}$ Significant difference compared with alcoholics at risk of WE (one criterion, $p<0.05)$.

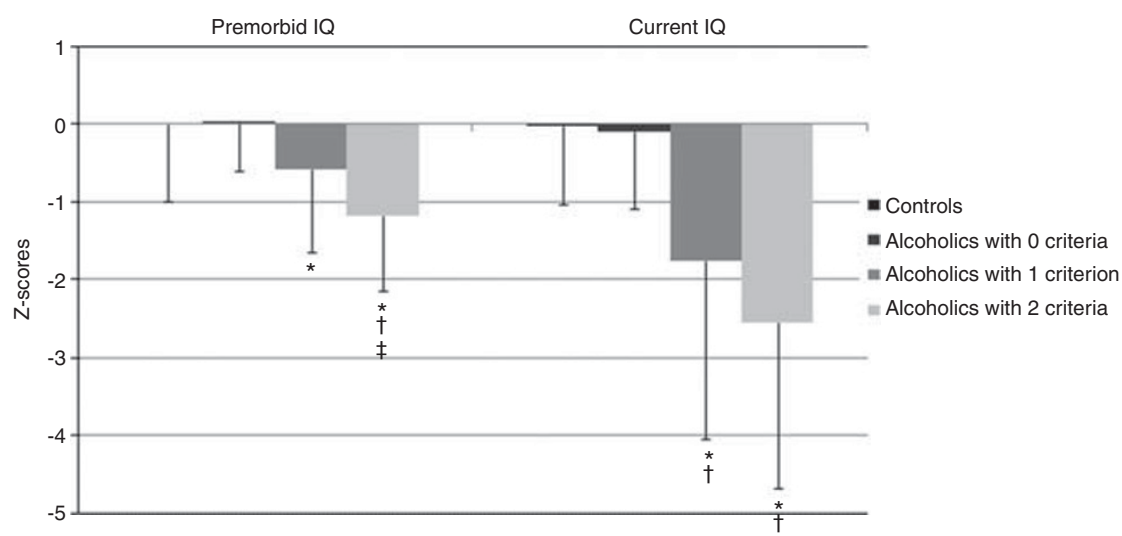

Figure 3 Premorbid and current IQ in the control group and the three subgroups of alcoholics (mean \pm SD). *Significant difference compared with controls $(p<0.05)$. "Significant difference compared with alcoholics who did not meet any criteria $(p<0.05)$. ${ }^{\ddagger}$ Significant difference compared with alcoholics at risk of WE (one criterion, $p<0.05$ ).

Alcoholics who did not meet any WE criteria did not differ from control subjects on any of the neuropsychological measures $(p>0.20$ in each case; Figure 2). Patients at risk for WE (one criterion) had lower IQ $(t(57)=3.58$, $p<0.001)$, memory performance $(t(89)=3.89, p \leqslant 0.001)$, visuoconstruction abilities $(t(68)=2.20, p=0.03)$, and processing speed $(t(68)=3.39, p=0.001)$ than controls and lower scores on tests of IQ $(t(39)=2.67, p=$ $0.01)$, memory $(t(40)=3.75, p<0.001)$, visuoconstruction $(t(45)=2.10, p=0.04)$, and processing speed $(t(44)=2.95$, $p=0.005)$ than alcoholics who did not meet any criteria. Relative to controls, patients with signs of WE (two or more criteria) also had lower IQ $(t(36)=5.32, p \leqslant 0.001)$, memory performance $(t(36)=4.00, \quad p \leqslant 0.001)$, and processing speed $(t(45)=6.05, p<0.001)$ and also performed worse on executive $(t(45)=3.46, p \leqslant 0.001)$, visuoconstruction $(t(45)=2.07, p=0.04)$, and upper limb motor $(t(45)=$ 2.39, $p=0.02$ ) tasks. Compared with alcoholics who did not meet any criteria, they had lower scores on tasks related to IQ $(t(18)=4.72, p \leqslant 0.001)$, memory $(t(18)=4.41$, $p \leqslant 0.001)$, processing speed $(t(21)=4.77, p<0.001)$, execu- tive functioning $(t(21)=3.33, p=0.003)$, visuoconstruction $(t(22)=2.39, p=0.03)$, and upper limb abilities $(t(21)=$ $2.38, p=0.03$ ) (Figure 2). Although alcoholics with signs of WE performed systematically worse than those at risk of WE, the only statistically significant difference was for processing speed $(t(39)=2.58, p=0.01)$. All these results were confirmed with non-parametric Mann-Whitney $U$-tests $(p \leqslant 0.05)$.

Premorbid and current IQ. Repeated measures ANOVA for the four groups (control group and three alcoholic subgroups) and two neuropsychological domains (premorbid and current IQ) revealed significant effects of group $(\mathrm{F}(3,75)=8.04 ; \quad p<0.001)$, neuropsychological domains $(\mathrm{F}(1,75)=6.00 ; p=0.02)$, and a tendency for an interaction $(\mathrm{F}(3,75)=2.40 ; p=0.08)$. When the controls were removed from the analysis, there was no longer a trend for the interaction $(\mathrm{F}(2,47)=0.66 ; p=0.52)$. Alcoholics who did not meet any WE criteria did not differ from control subjects on any of the measures $(p>0.80$ in each case; Figure 3). Patients at risk for or with signs of WE were 


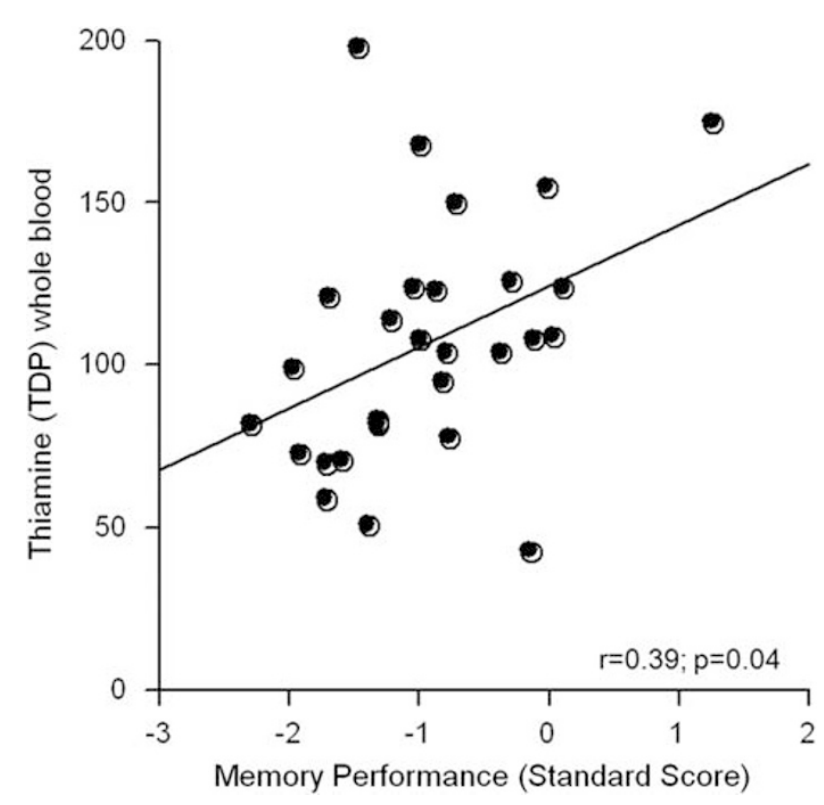

Figure 4 Relationships between thiamine measures and memory performance in the total group of alcoholics* (*bloods were collected from $N=28$ alcoholics).

impaired on the two IQ measures compared with control subjects $(p<0.05$ in each case). Compared with alcoholics who did not meet any WE criteria, those at risk for WE were impaired on the current IQ measure $(p=0.04$; with a tendency for impairment on the premorbid IQ, $p=0.08$ ) and those with signs of WE were impaired on both IQ measures $(p<0.01)$. Finally, alcoholics with signs of WE were impaired on the premorbid IQ $(p=0.04)$ compared with those at risk for WE.

\section{Relationships between Neuropsychological Test Scores and TDP Levels}

The levels of TDP did not differ significantly between the 22 controls and 28 alcoholics for whom the whole blood analysis had been conducted. Although alcoholics classified as having had dietary deficiencies had lower TDP levels $(101.62 \pm 27.50, N=21)$ than those without this history $(123.29 \pm 59.10, N=7)$, the difference was not significant $(t(26)=1.33, p=0.19)$. In alcoholics, TDP levels did not correlate with length of sobriety, lifetime alcohol consumption, AUDIT, or SADDQ scores ( $p>0.15$ in all cases).

Multiple regressions tested relations between TDP levels and the neuropsychological tests showing lower scores in alcoholics than controls and commonly affected in uncomplicated alcoholics: memory, executive function, visuoconstruction, and lower limb ataxia. In the alcoholic group, these neuropsychological variables accounted for $25 \%$ of TDP variance, but a selective relation was identified between poorer memory scores and lower TDP levels $(\beta=28.94, p=0.02)$ over and above relations with the three other test scores (Figure 4). In alcoholics with a history of nutritional deficiency, further analysis revealed that TDP levels were related to IQ $(\rho=0.44, p=0.04)$ and memory $(\rho=0.57, p<0.01)$ and showed a tendency to correlate with executive functions $(\rho=0.38, p=0.09)$. In contrast, none of the neuropsychological scores correlated with the TDP measures ( $p>0.30$ in all cases) in alcoholics without a history of nutritional deficiency.

\section{DISCUSSION}

Application of the criteria of Caine et al (1997) revealed that $16 \%$ of the 'uncomplicated alcoholics' in this sample had signs of WE (ie met two criteria), which is in agreement with the prevalence rates reported postmortem in this population (12-14\%; Harper et al, 1986), and indicates a higher than expected potential occult incidence of this neuropsychiatric disorder in alcoholics without apparent complications. Half of the alcoholic group met criteria for dietary deficiency and had lower TDP levels than those without a history of nutritional deficiency even though the two groups did not significantly differ on TDP level. This finding suggests that these two ways of measuring potential dietary deficiency may reflect two different but related phenomena: the nutritional status at the time of the blood test and a history of withholding meals while drinking. The use of biomarkers complements the taking of a comprehensive history, which remains essential because there is currently no established threshold level of TDP above which patients would be safe from developing brain damage (Thomson et al, 2010). Our preliminary findings indicate that current TDP concentrations are not influenced by the length of sobriety, the severity of alcohol consumption or dependence, or age.

Ataxia was also frequently observed among alcoholics (Sullivan et al, 2010). That only two alcoholics had nystagmus and none had mild-cognitive impairment may reflect the rarity of certain criteria (Caine et al, 1997; Harper et al, 1986; Naidoo et al, 1991), suboptimal measurements used herein, or the resolution of these signs following only a short period of sobriety. Extending earlier work (Caine et al, 1997), this study also shows that more than half of the 'uncomplicated alcoholics' met one criterion and may, therefore, be considered at risk for WE. These findings encourage the use of the Caine et al criteria in clinical settings for detection, prevention, and treatment of alcoholics at risk for or with signs of alcohol-related neuropsychiatric complications before the development of irreversible and debilitating damage such as KS. These criteria could be used together with the clinical protocols for diagnosis and treatment developed by Thomson and colleagues (Thomson et al, 2002; Thomson and Marshall, 2006). Given the high prevalence of preclinical signs of WE in uncomplicated alcoholics and the low reports of anaphylactic reaction with thiamine supplementation (for review, see Thomson et al, 2002), such treatment could be proposed not only to alcoholics with ostensible clinical signs of WE, but to all alcoholics. The addition of thiamine to food products has been another established method of delivery of thiamine for many years, particularly the enrichment of flour (Harper et al, 1998).

Our neuropsychological results comport with previous investigations showing that, when analyzed as a group, alcoholics have lower performance than controls on scores reflecting the average of verbal and performance IQ (Tedstone and Coyle, 2004), memory (Pitel et al, 2007a), and processing speed tasks (Pitel et al, 2007b). However, 
whole group analyses cannot address the heterogeneity in the profiles of cognitive and motor performance observed within a sample of alcoholics. By conducting subgroup analysis, this study supports previous individual analysis (Pitel et al, 2008), indicating that in some patients, alcoholism does not affect cognitive and motor functioning, while in others, severe alcohol-related neuropsychological impairments occur.

Heterogeneity in the patterns of neuropsychological performance in the alcoholics examined herein was observed. Comparison between subgroups of alcoholics classified according to the WE criteria of Caine et al revealed a graded effect in the cognitive and motor performance of the alcoholic subgroups, with those not meeting any criteria performing at control levels, those at risk for WE showing mild-to-moderate deficits on some of the functional domains examined, and those with signs of WE showing the most severe deficits on each of the domains. These graded effects suggest, first, that the presence of signs of WE explains, at least partially, the heterogeneity of alcoholism-related cognitive and motor deficits. Second, because alcoholics at risk for WE performed worse than controls or alcoholics not meeting any WE criteria on tests of IQ, memory, visuoconstruction, and processing speed, these domains may be particularly vulnerable to the effects of alcohol. This study also provides preliminary correlational evidence that memory performance in alcoholics is especially related to levels of the biologically active form of thiamine, indicating that this functional domain of memory may be particularly sensitive to impairments in the levels of thiamine even when in the normal range. Interestingly, we found relationships between neuropsychological performance and TDP concentrations in the group of alcoholics taken as a whole and in those with a history of nutritional deficiency, but not in those without such history. Alcoholics with a history of nutritional deficiency, who present with slightly lower TDP levels than those without such history may be more susceptible to the combined effects of alcohol and thiamine decreases, or lower TDP levels may be an enduring marker for previous bouts of nutritional insufficiencies. Third, because the alcoholic subgroups did not differ with respect to age, sex, lifetime alcohol intake, or length of sobriety, these factors are unlikely to explain why alcoholics differ in the number of criteria met or the extent of functional impairment. Higher education in alcoholics with no WE criteria suggests that education might be considered as protective against alcohol-related neuropsychiatric disorders, similar, perhaps, to what has been described in other disorders such as Alzheimer's disease (Querbes et al, 2009). However, differences observed between alcoholic subgroups in neuropsychological performance endured after controlling for education, indicating that this factor did not explain the graded effect of cognitive and motor deficits. The analysis of IQ scores comports with these findings because current IQ was not disproportionately different from premorbid IQ in the subgroups of alcoholics. Finally, other factors not investigated in this study, such as the individual genetically determined vulnerability to the combined effects of alcohol and thiamine deficiency, are potential contributors to heterogeneity in the expression of alcoholism-related neuropsychological compromise.
These conclusions are based on a limited sample and require replication with larger and more diverse group including notably more female alcoholics. Nonetheless, this initial study provides promising evidence for the utility of Caine et al criteria in identifying alcoholics at risk for WE. Use of such retrospective criteria combined with current examination and thiamine testing (TDP in whole blood) could be instrumental in overcoming certain challenges of in vivo human study, including questionable reliability of some clinical variables such as the estimation of lifetime alcohol consumption or the nutritional history, especially in alcoholics with neuropsychological deficits.

\section{ACKNOWLEDGEMENTS}

This research was supported by grants from the US National Institute on Alcohol Abuse and Alcoholism (AA010723, AA017923, AA017168, AA005965). The funders had no role in study design, data collection and analysis, decision to publish, or preparation of the manuscript.

\section{DISCLOSURE}

The authors declare no conflict of interest.

\section{REFERENCES}

Baker KG, Harding AJ, Halliday GM, Kril JJ, Harper CG (1999). Neuronal loss in functional zones of the cerebellum of chronic alcoholics with and without Wernicke's encephalopathy. Neuroscience 91: 429-438.

Caine D, Halliday GM, Kril JJ, Harper CG (1997). Operational criteria for the classification of chronic alcoholics: identification of Wernicke's encephalopathy. J Neurol Neurosurg Psychiatry 62: 51-60.

Cargiulo T (2007). Understanding the health impact of alcohol dependence. Am J Health Syst Pharm 64(5 Suppl 3): S5-11.

Corkin S, Growdon JH, Sullivan EV, Nissen MJ, Huff FJ (1986). Assessing treatment effects from a neuropsychological perspective. In: L Poon (ed). Handbook of Clinical Memory Assessment in Older Adults. American Psychological Association: Washington DC. pp 156-167.

Dawson LK, Grant I (2000). Alcoholics' initial organizational and problem-solving skills predict learning and memory performance on the Rey-Osterrieth Complex Figure. J Int Neuropsychol Soc 6: 12-19.

Day E, Bentham P, Callaghan R (2008). Thiamine for WernickeKorsakoff Syndrome in People at Risk from Alcohol Abuse. Wiley: Chichester.

Fein G, Landman B (2005). Treated and treatment-naive alcoholics come from different populations. Alcohol 36: 19-26.

First MB, Spitzer RL, Gibbon M, Williams JBW (1995). Structured Clinical Interview for DSM-IV Axis I Disorders. Biometrics Research Department, New York State Psychiatric Institute: New York.

Fregly AR, Smith MJ, Graybiel A (1973). Revised normative standards of performance of men on a quantitative ataxia test battery. Acta Otolaryngol 75: 10-16.

Guerrini I, Thomson AD, Gurling HM (2009). Molecular genetics of alcohol-related brain damage. Alcohol Alcohol 44: 166-170.

Harper CG, Sheedy DL, Lara AI, Garrick TM, Hilton JM, Raisanen J (1998). Prevalence of Wernicke-Korsakoff syndrome in Australia: has thiamine fortification made a difference? Med J Aust 168: 542-545. 
Harper C (2009). The neuropathology of alcohol-related brain damage. Alcohol Alcohol 44: 136-140.

Harper C (2006). Thiamine (vitamin B1) deficiency and associated brain damage is still common throughout the world and prevention is simple and safe!. Eur J Neurol 13: 1078-1082.

Harper CG, Giles M, Finlay-Jones R (1986). Clinical signs in the Wernicke-Korsakoff complex: a retrospective analysis of 131 cases diagnosed at necropsy. J Neurol Neurosurg Psychiatry 49: 341-345.

Kopelman MD, Thomson AD, Guerrini I, Marshall EJ (2009). The Korsakoff syndrome: clinical aspects, psychology and treatment. Alcohol Alcohol 44: 148-154.

Lezak MD (1995). Neuropsychological Assessment, 3rd edn. Oxford University Press: New York.

Mancinelli R, Ceccanti M, Guiducci MS, Sasso GF, Sebastiani G, Attilia ML et al (2003). Simultaneous liquid chromatographic assessment of thiamine, thiamine monophosphate and thiamine diphosphate in human erythrocytes: a study on alcoholics. J Chromatogr B Analyt Technol Biomed Life Sci 789: 355-363.

Matthews CG, Kleve H (1964). Instruction Manual for the Adult. Neuropsychology Test Battery. University of Wisconsin Medical School: Madison, WI.

Mattis S (1988). Dementia Rating Scale (DRS) Professional Manual. Psychological Assessment Resources, Inc: Odessa, FL.

Naidoo DP, Bramdev A, Cooper K (1991). Wernicke's encephalopathy and alcohol-related disease. Postgrad Med J 67: 978-981.

Nelson HE (1982). The National Adult Reading Test (NART). Nelson Publishing Company: Windsor, Canada.

Noel X, Van der Linden M, Schmidt N, Sferrazza R, Hanak C, Le Bon O et al (2001). Supervisory attentional system in nonamnesic alcoholic men. Arch Gen Psychiatry 58: 1152-1158.

Osterrieth P, Rey A (1944). Le test de copie d'une figure complex. Archives de Psychologie 30: 205-221.

Parsons OA (1998). Neurocognitive deficits in alcoholics and social drinkers: a continuum? Alcohol Clin Exp Res 22: 954-961.

Pitel AL, Beaunieux H, Witkowski T, Vabret F, de la Sayette V, Viader F et al (2008). Episodic and working memory deficits in alcoholic Korsakoff patients: the continuity theory revisited. Alcohol Clin Exp Res 32: 1229-1241.

Pitel AL, Beaunieux H, Witkowski T, Vabret F, Guillery-Girard B, Quinette $\mathrm{P}$ et al (2007a). Genuine episodic memory deficits and executive dysfunctions in alcoholic subjects early in abstinence. Alcohol Clin Exp Res 31: 1169-1178.

Pitel AL, Witkowski T, Vabret F, Guillery-Girard B, Desgranges B, Eustache $\mathrm{F}$ et al (2007b). Effect of episodic and working memory impairments on semantic and cognitive procedural learning at alcohol treatment entry. Alcohol Clin Exp Res 31: 238-248.

Querbes O, Aubry F, Pariente J, Lotterie JA, Demonet JF, Duret V et al (2009). Early diagnosis of Alzheimer's disease using cortical thickness: impact of cognitive reserve. Brain 132(Pt 8): 2036-2047.

Raistrick D, Dunbar G, Davidson R (1983). Development of a questionnaire to measure alcohol dependence. $\mathrm{Br} J$ Addict 78: 89-95.

Rey A (1942). L'examen psychologique dans les cas d'encephalopathie traumatique. Arch Psychol 28: 286.
Ryback RS (1971). The continuum and specificity of the effects of alcohol on memory. A review. Q J Stud Alcohol 32: 995-1016.

Saunders JB, Aasland OG, Babor TF, de la Fuente JR, Grant M (1993). Development of the Alcohol Use Disorders Identification Test (AUDIT): WHO Collaborative Project on Early Detection of Persons with Harmful Alcohol Consumption-II. Addiction 88: 791-804.

Sechi G, Serra A (2007). Wernicke's encephalopathy: new clinical settings and recent advances in diagnosis and management. Lancet Neurol 6: 442-455.

Skinner HA (1982). Development and Validation of a Lifetime Alcohol Consumption Assessment Procedure. Addiction Research Foundation: Toronto, Canada.

Sullivan EV, Deshmukh A, Desmond JE, Lim KO, Pfefferbaum A (2000a). Cerebellar volume decline in normal aging, alcoholism, and Korsakoffs syndrome: relation to ataxia. Neuropsychology 14: 341-352.

Sullivan EV, Pfefferbaum A (2009). Neuroimaging of the Wernicke-Korsakoff syndrome. Alcohol Alcohol 44: 155-165.

Sullivan EV, Rohlfing T, Pfefferbaum A (2010). Pontocerebellar volume deficits and ataxia in alcoholic men and women: no evidence for 'telescoping'. Psychopharmacology (Berl) 208: 279-290.

Sullivan EV, Rosenbloom MJ, Pfefferbaum A (2000b). Pattern of motor and cognitive deficits in detoxified alcoholic men. Alcohol Clin Exp Res 24: 611-621.

Tedstone D, Coyle K (2004). Cognitive impairments in sober alcoholics: performance on selective and divided attention tasks. Drug Alcohol Depend 75: 277-286.

Thomson AD (2000). Mechanisms of vitamin deficiency in chronic alcohol misusers and the development of the Wernicke-Korsakoff syndrome. Alcohol Alcohol Suppl 35: 2-7.

Thomson AD, Cook CC, Touquet R, Henry JA (2002). The Royal College of Physicians report on alcohol: guidelines for managing Wernicke's encephalopathy in the accident and Emergency Department. Alcohol Alcohol 37: 513-521.

Thomson AD, Jeyasingham MD, Pratt OE, Shaw GK (1987). Nutrition and alcoholic encephalopathies. Acta Med Scand Suppl 717: 55-65.

Thomson AD, Marshall EJ (2006). The treatment of patients at risk of developing Wernicke's encephalopathy in the community. Alcohol Alcohol 41: 159-167.

Thomson AD, Marshall EJ, Guerrini I (2010). Biomarkers for detecting thiamine deficiency-improving confidence and taking a comprehensive history are also important. Alcohol Alcohol 45: 213 .

Torvik A, Lindboe CF, Rogde S (1982). Brain lesions in alcoholics. A neuropathological study with clinical correlations. J Neurol Sci 56: $233-248$.

Wechsler D (1981). Wechsler Adult Intelligence Scale-Revised. The Psychological Corporation: San Antonio, TX.

Wechsler D (2001). Weschler Adult Intelligence Scale. EAP: Paris. Wechsler DT (1987). The Wechsler Memory Scale-Revised. Psychological Corporation: San Antonio.

Wernicke C (1881). Lehrbuch der Gehirnkrankheiten fur Artze und Studierende. Fischer: Berlin. 